Debate piece for ANZJP

\title{
How effective are 'headspace' youth mental health services?
}

Anthony F Jorm

Centre for Mental Health, Melbourne School of Population and Global Health, University of Melbourne, Australia

\section{Corresponding author:}

Anthony F Jorm, Melbourne School of Population and Global Health, University of Melbourne, VIC 3010, Australia.

Email: ajorm@unimelb.edu.au

Key words: youth, adolescents, psychological therapy, early intervention, depression, anxiety 
There is a high prevalence of mental disorders in Australian youth, but many of those affected do not seek professional help. In response to this treatment gap, the Australian government set up a National Youth Mental Health Foundation, 'headspace', in 2006. This initiative involved the setting up of an initial 10 enhanced primary care services for young people aged 12-15 years, offering early intervention for mental health and substance use problems, as well as physical health care and vocational services. These services were designed to be 'youth-friendly' and to overcome the stigma attached to traditional services. They also aimed to break down the age divide between adolescent and adult services. This headspace model has been enthusiastically supported by successive Australian governments, with the number of headspace centres funded due to expand to 100 by 2016. Other countries have also begun to adopt the model.

However, it is important to note that this expansion has occurred in the absence of any evidence that headspace services are actually effective in improving youth mental health.

While there have been descriptive studies of headspace clients, outcome data has been lacking until recently. It is therefore very welcome to see the publication of two papers by an evaluation team working for headspace (Rickwood et al. 2015a, 2015b), which give the most comprehensive data to date.

\section{What was found?}

Rickwood and colleagues $(2015 a, 2015 b)$ examined data from a large sample of clients, using the headspace minimum dataset. They found that most clients (73\%) sought help for 'mental health and behaviour' problems, primarily anxiety and depressive symptoms. The most common type of service received by clients was cognitive-behaviour therapy, with smaller proportions receiving other types of psychological therapy. Remarkably, data on medication use was not recorded.

Symptom severity was assessed using the K10 scale, which measures selfreported psychological distress on a 40-point scale. The initial mean score was very high, indicating a need for intervention. However, improvement from first to last assessment was a mean of only 3 points (Rickwood et al., 2015a). When K10 scores were classified according to whether they showed 'significant change' (defined as $\geq 0.5$ of a standard deviation unit), only $36 \%$ of clients improved, $51 \%$ showed no change and $13 \%$ deteriorated. When clients who initially scored in the clinical range on the K10 were examined, only $21 \%$ of them showed 'clinically significant change' by crossing the threshold that marked transition from a clinical to a non-clinical population.

\section{Could the changes simply be spontaneous remission?}

While more clients improved than worsened, Rickwood and colleagues (2015a) appropriately noted that "the absence of a control group...means that changes in scores reported cannot be attributed to headspace care". It is well known that 
people with depression and anxiety disorders tend to improve even without intervention, so some or most of the improvements they described could be due to spontaneous remission. Some indication of what might be expected from spontaneous remission comes from two meta-analyses of improvement in untreated depressed patients.

The first meta-analysis involved 10 studies, including two that involved adolescents (Rutherford et al., 2012). It found a standardized mean difference of 0.5 over a mean duration of 10 weeks. By comparison, the standardized mean difference on the K10 for headspace clients was approximately 0.33 at last assessment and 0.67 at 3-month follow-up.

The second meta-analysis examined 'remission' in untreated depression, with the remission criteria being similar to Rickwood and colleagues' (2015a) definition of 'clinically significant improvement' (Whiteford et al., 2013). This meta-analysis found that $23 \%$ of adults remitted at 3 months, with a higher remission rate (not given in their paper) in children and adolescents. By comparison, only $21 \%$ of headspace clients showed clinically significant improvement by their last assessment.

It can be concluded that the improvements seen in headspace clients are similar to those seen in untreated cases and it is would seem that the services provided may have had little or no effect.

\section{Why were the changes not greater?}

Given that cognitive-behaviour therapy and other psychological therapies are known to be effective for depression and anxiety disorders in randomized controlled trials, we must ask why headspace clients did not in fact show greater improvements. The answer may be that they did not receive a sufficient 'dose' of treatment. A recent study of quality of mental health treatment in Australia defined 'minimally adequate treatment' for psychological therapy as involving 6 or more sessions of 30 minutes duration or longer (Harris et al., 2015).

Applying these criteria to the data of Rickwood et al. (2015b), only $28 \%$ of clients presenting for mental health and behaviour problems received 'minimally adequate treatment'. Many fell well short, with 45\% receiving only 1-2 sessions.

It could be argued that these clients only required 1-2 sessions. But if that were the case, then these clients should have experienced clinically significant improvement in their symptoms, indicating that they did not require further treatment. However, the published data do not support this.

Given that headspace services were specifically designed to appeal to young people and to reduce the stigma of seeking professional help, the level of engagement observed is disappointing. There is a need to compare the level of engagement in headspace services with that in traditional adolescent and adult services to see if they do any better. 


\section{Where to from here?}

There is no doubt that the high prevalence of mental health problems in young people requires action. However, unless it can be clearly demonstrated that headspace is improving the mental health of its clients, it would be unwise to invest further in this model. This is because there is a substantial opportunity cost in tying up resources that could be used to explore alternative approaches that might be more effective. The Australian Government has commissioned an independent evaluation of headspace services, which has yet to be released. It remains to be seen whether or not it will provide the convincing evidence that is needed.

The expansion of headspace services is an example of a phenomenon we see too often in the mental health sector, with innovations that have an appealing rationale rolled out nationally in advance of proper evaluation. Recently, the National Mental Health Commission recommended major reforms of the sector and the Australian Government is currently considering the implementation of these. It is to be hoped that any reforms implemented will be properly trialled and their quality and impact assessed before, rather than after, any national rollout.

\section{Funding}

The author is supported by an NHMRC Fellowship.

\section{Declaration of interest}

The author has collaborated with headspace staff, including on research projects involving headspace clients as participants. He was formerly associated with Orygen Youth Health Research Centre, which runs several headspace services.

\section{References}

Harris MG, Hobbs MJ, Burgess PM, et al. (2015) Frequency and quality of mental health treatment for affective and anxiety disorders among Australian adults. Medical Journal of Australia 202: 185-190.

Rickwood DJ, Mazzer KR, Telford NR, et al. (2015a). Changes in psychological distress and psychosocial functioning in young people visiting headspace centres for mental health problems. Medical Journal of Australia 202; 537542.

Rickwood DJ, Telford NR, Mazzer KR, et al. (2015b). The services provided to young people through the headspace centres across Australia. Medical Journal of Australia 202; 533-536.

Rutherford BR, Mori S, Sneed JR, et al. (2012) Contribution of spontaneous improvement to placebo response in depression: a meta-analytic review. Journal of Psychiatric Research 46: 697-702.

Whiteford HA, Harris MG, McKeon G, et al. (2013) Estimating remission from untreated major depression: a systematic review and meta-analysis. Psychological Medicine 43: 1569-1585. 


\section{University Library}

\section{- M M I N E R VA A gateway to Melbourne's research publications}

Minerva Access is the Institutional Repository of The University of Melbourne

Author/s:

Jorm, AF

Title:

How effective are "headspace' youth mental health services?

Date:

2015-10-01

Citation:

Jorm, A. F. (2015). How effective are "headspace' youth mental health services?

AUSTRALIAN AND NEW ZEALAND JOURNAL OF PSYCHIATRY, 49 (10), pp.861-862. https://doi.org/10.1177/0004867415608003.

Persistent Link:

http://hdl.handle.net/11343/91134 